\title{
A novel MRI-based score assessing trabecular bone quality to predict vertebral compression fractures in patients with spinal metastasis
}

\author{
Jeff Ehresman, BS, ${ }^{1}$ Andrew Schilling, AB, ${ }^{1}$ Zach Pennington, BS, ${ }^{1}$ Chengcheng Gui, BS, ${ }^{2}$ \\ Xuguang Chen, MD, PhD, ${ }^{2}$ Daniel Lubelski, MD, ${ }^{1}$ A. Karim Ahmed, BS, ${ }^{1}$ Ethan Cottrill, BS, ${ }^{1}$ \\ Majid Khan, MD, ${ }^{3}$ Kristin J. Redmond, MD, MPH, ${ }^{2}$ and Daniel M. Sciubba, MD ${ }^{1}$ \\ Departments of ${ }^{1}$ Neurosurgery, ${ }^{2}$ Radiation Oncology, and ${ }^{3}$ Radiology, The Johns Hopkins University School of Medicine,
Baltimore, Maryland
}

\begin{abstract}
OBJECTIVE Vertebral compression fractures (VCFs) in patients with spinal metastasis can lead to destabilization and often carry a high risk profile. It is therefore important to have tools that enable providers to predict the occurrence of new VCFs. The most widely used tool for bone quality assessment, dual-energy x-ray absorptiometry (DXA), is not often available at a patient's initial presentation and has limited sensitivity. While the Spinal Instability Neoplastic Score (SINS) has been associated with VCFs, it does not take patients' baseline bone quality into consideration. To address this, the authors sought to develop an MRI-based scoring system to estimate trabecular vertebral bone quality (VBQ) and to assess this system's ability to predict the occurrence of new VCFs in patients with spinal metastasis.

METHODS Cases of adult patients with a diagnosis of spinal metastasis, who had undergone stereotactic body radiation therapy (SBRT) to the spine or neurosurgical intervention at a single institution between 2012 and 2019, were retrospectively reviewed. The novel VBQ score was calculated for each patient by dividing the median signal intensity of the L1-4 vertebral bodies by the signal intensity of cerebrospinal fluid (CSF). Multivariable logistic regression analysis was used to identify associations of demographic, clinical, and radiological data with new VCFs.
\end{abstract}

RESULTS Among the 105 patients included in this study, 56 patients received a diagnosis of a new VCF and 49 did not. On univariable analysis, the factors associated with new VCFs were smoking status, steroid use longer than 3 months, the SINS, and the novel scoring system-the VBQ score. On multivariable analysis, only the SINS and VBQ Score were significant predictors of new VCFs and, when combined, had a predictive accuracy of $89 \%$.

CONCLUSIONS As a measure of bone quality, the novel VBQ score significantly predicted the occurrence of new VCFs in patients with spinal metastases independent of the SINS. This suggests that baseline bone quality is a crucial factor that requires assessment when evaluating these patients' conditions and that the VBQ score is a novel and simple MRIbased measure to accomplish this.

https://thejns.org/doi/abs/10.3171/2019.9.SPINE19954

KEYWORDS vertebral bone quality; magnetic resonance imaging; compression fracture; bone; metastasis; trabecular bone; oncology

$\mathrm{S}$ PINAL column metastases are common among patient with disseminated cancer. Although, in most cases, these metastases are minimally symptomatic or asymptomatic, a nontrivial proportion serve as mechanical pain generators secondary to the destruction of the affected vertebrae. Equally as important, as the lesion enlarges, it reduces the yield strength of affected vertebrae and increases the risk of vertebral compression fracture (VCF). ${ }^{5,6} \mathrm{~A}$ VCF can destabilize the vertebral column and thus requires surgical intervention, which generally carries a high risk profile in this frail and medically complex patient population. ${ }^{23}$

ABBREVIATIONS AUC = area under the receiver operating characteristic curve; BMD = bone mineral density; CSF = cerebrospinal fluid; DXA = dual-energy X-ray absorptiometry; ICC = intraclass correlation coefficient; NSAID = nonsteroidal antiinflammatory drug; ROI = region of interest; SBRT = stereotactic body radiation therapy; SINS = Spinal Instability Neoplastic Score; VBQ = vertebral bone quality; VCF = vertebral compression fracture.

SUBMITTED August 14, 2019. ACCEPTED September 30, 2019.

INCLUDE WHEN CITING Published online December 20, 2019; DOI: 10.3171/2019.9.SPINE19954. 
Despite the obvious benefits of being able to accurately predict VCF among patients with spinal cancer, the ability of physicians to do so in clinical practice is limited. One tool that has gained popularity is the Spinal Instability Neoplastic Score (SINS), a multidimensional scoring system developed by the Spinal Oncology Study Group to grade the level of instability associated with a metastasisburdened vertebra.? The SINS has been demonstrated to predict the occurrence of a VCF; 22 however, it suffers from the fact that it neither includes systemic factors, such as bone mineral density (BMD), nor is designed to give an estimate of the probability of fracture. Both endpoints, however, are addressed by the Fracture Risk Assessment Tool (FRAX), designed by the World Health Organization to predict the 10 -year risk of major osteoporotic fracture..$^{10}$ That tool focuses on the demographics, previous treatments, and BMDs of patients; however, it does not include oncology-specific risk factors.

It is intuitive that a patient with poor bone quality may be at an increased risk for VCF. While a few studies have found a low BMD to be associated with an increased risk for osteoporotic $\mathrm{VCF},{ }^{12}$ this relationship has not been studied in patients with spinal metastases. One reason for the lack of study may be that many patients present with metastasis to the spine before they reach the age of 65 years, which is the current advised age of dual-energy x-ray absorptiometry (DXA) screening. ${ }^{4}$ Therefore, many patients with metastatic spine disease do not have DXA scans readily available, leading to additional time and costs for patients. To improve measures that predict pathological VCFs, one needs to be able to evaluate bone quality from image modalities commonly acquired within the population of patients presenting with spinal metastasis, such as MRI or CT.1,21 One opportunity stems from the Coronary Artery Risk Development in Young Adults (CARDIA) trial, which found lower cancellous bone fat content on T1 imaging to be negatively correlated with BMD. ${ }^{21}$ By taking advantage of patients' previous MRI studies to identify those at high risk of VCF, providers would have the ability to treat poor bone quality with pro-osteogenic therapies in addition to considering cement-augmented techniques in the surgical population.

In an attempt to better predict VCFs in this patient population, the aims of this study were 1) to develop an easy-to-use MRI scoring system to estimate trabecular vertebral bone quality (VBQ) and 2) to assess the ability of this novel scoring method and other risk factors to predict the occurrence of new VCFs in patients with spinal metastases.

\section{Methods \\ Study Population}

After we had obtained approval from The Johns Hopkins University institutional review board, we queried the medical records of all patients who had undergone either radiation therapy or surgical intervention at Johns Hopkins Hospital between January 2012 and June 2019. Patients were included if they were at least 18 years old, had a diagnosis of spinal metastasis, had undergone T1weighted non-contrast-enhanced MRI of the lumbar spine without previous lumbar instrumentation, had presented to Johns Hopkins Hospital with no more than one previous VCF, and had attended follow-up examinations for at least 6 months after the diagnosis of spine metastasis. Of the 80 patients in whom stereotactic body radiation therapy (SBRT) had been performed using a robotic system and T1-weighted MR images of the lumbar spine had been obtained, 54 met the inclusion criteria for this study. The imaging protocol for these patients included repeated MRI every 3 months for the 1st year of diagnosis, every 4 months for the 2 nd year, and every 6 months thereafter. Of the 210 patients in whom surgery had been performed and T1-weighted MR images of the lumbar spine had been obtained, 51 met the total inclusion criteria, with the majority being excluded due to early surgical intervention at the index level, before the lesion could be monitored for at least 6 months. The imaging protocol for these patients included preoperative and 3-month postoperative MRI, followed by repeated imaging every 6 months for the entire duration of follow-up. Patients were also excluded if they initially presented with multiple compression fractures or a diagnosis of infection. This led to a total of 105 patients being included in this study.

\section{Recorded Variables}

We retrospectively reviewed the medical records and gathered patient demographics (age, sex, and body mass index); smoking status; and use of alcohol, steroid medications, and nonsteroidal antiinflammatory drugs (NSAIDs). We included each individual patient's comorbidities as well as their Charlson Comorbidity Index score, baseline functional status (Karnofsky Performance Scale score), Eastern Cooperative Oncology Group Performance Status grade, serum lab values, and previous treatments that include vertebroplasty/kyphoplasty, denosumab treatment, bisphosphonate treatment, calcium/vitamin D supplementation, cumulative dose of radiation to index vertebrae, and overall systemic treatment duration. Last, we gathered all relevant radiological data in order to record the SINS, the presence of VCFs, and the MRI values needed to produce the VBQ score.

\section{MR Image Analysis}

MRI examinations were performed using both 1.5-T and 3-T images from multiple brands of MRI machines used at our institution, namely Philips Healthcare, General Electric (GE) Healthcare, Toshiba Corp., and Siemens Healthineers USA, Inc. All analyzed images were T1 weighted. Fluid attenuation inversion recovery, inversion recovery, and postcontrast images were excluded. ${ }^{1}$ To ensure that the brand of MR system and tesla setting were not potential confounders, values from each MR system and tesla setting were compared and are found in Supplemental Table 1. Calculation of the VBQ score was performed as shown in Fig. 1. A region of interest (ROI) was placed in each of the L1 through L4 vertebral bodies to calculate the average signal intensity of the designated area (labeled as AV in Fig. 1). The ROIs excluded cortical bone by placing a concentric ROI approximately $0.3 \mathrm{~cm}$ from the perimeter of the vertebral body. The ROI also ex- 


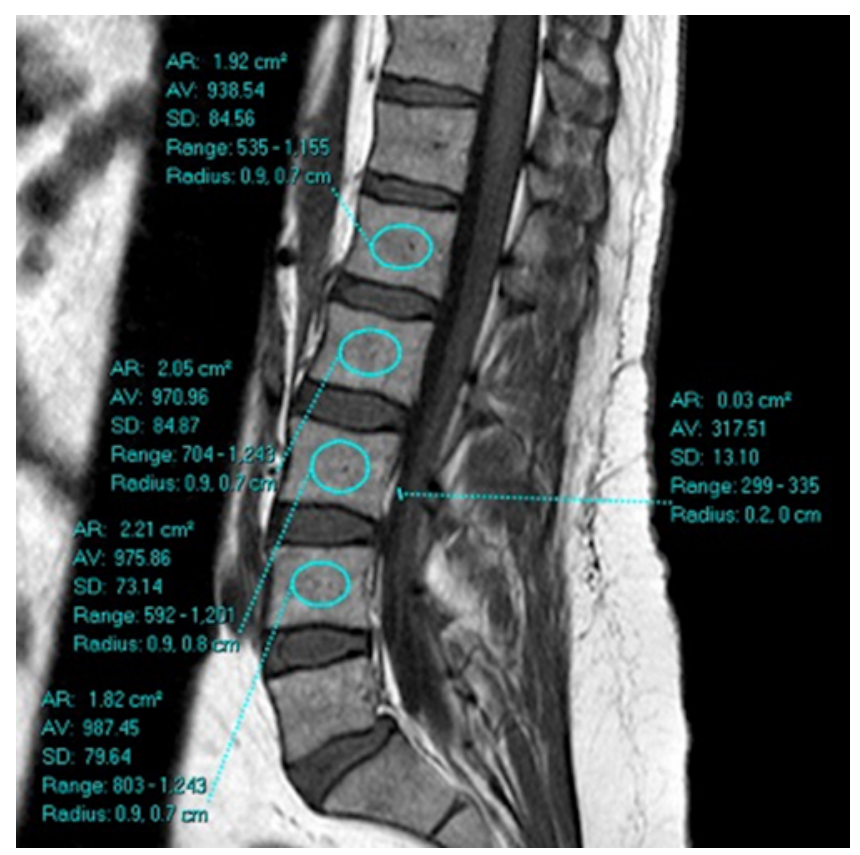

FIG. 1. Non-contrast-enhanced T1-weighted MR image detailing the ROls (circles) that are measured to calculate the VBQ score. AR = area of the ROI; $A V=$ average signal intensity of the ROI. Figure is available in color online only.

cluded focal lesions (metastatic lesions or hemangiomas) and the posterior venous plexus. If the MRI reader was unable to obtain an ROI by using the midsagittal image due to these obstructing structures, a parasagittal image was used instead. Furthermore, if a lumbar vertebral body had no available area of bone with which to measure signal intensity (i.e., body collapse or metastasis), then no ROI was recorded from the vertebrae. For example, if the vertebral body of L2 had wide metastatic involvement on MRI, this body was excluded and only values from L1, L3, and L4 would be obtained. The signal intensity of cerebrospinal fluid (CSF) was measured at the L3 level, also shown in Fig. 1. When this was not possible due to obstruction (e.g., spinal stenosis at L3), CSF at the level of L2 or L4 was used. All images were assessed and analyzed using Vue PACs software (Carestream).

\section{Statistical Analysis}

The novel VBQ score was calculated by two independent researchers as follows: the signal intensity (SI) of the medullary bone of the L1-4 vertebrae was obtained by placing the ROI over the vertebral body as shown in Fig. 1. To normalize the large variation of signal intensities between brands of MRI machines, CSF at the level of the L3 vertebra was used as an internal control for each image. Once the SI was discovered for each L1-4 vertebral body, the median of these values was divided by the SI of the CSF.

$$
\text { VBQ score }=\mathrm{SI}_{\mathrm{L} 1-4} / \mathrm{SI}_{\mathrm{CSF}}
$$

This value is the VBQ score, which is a direct measure of the hyperintensity of the lumbar vertebral bone marrow. As bone marrow adipose tissue appears hyperintense on T1-weighted MR images, ${ }^{18}$ it is likely that the VBQ score is a direct measure of the intravertebral fat fraction, which has been shown to be negatively correlated with the amount of trabecular bone. ${ }^{11,25}$ To create a binary VBQ score, a simplified cutoff value was established by calculating the mean VBQ score between the two outcome groups and rounding to the nearest integer. Both the continuous VBQ score and the categorial VBQ score (greater or lesser than the cutoff) were included in the analyses. Interrater reliability was assessed using the intraclass correlation coefficient (ICC).

The SINS was calculated as previously described? Briefly, the criteria include six components, with five being radiological measures. These include tumor location, quality of pain, type of bone lesion, radiographic spine alignment, degree of vertebral body collapse, and posterolateral involvement. These components were obtained through an analysis of MR images in addition to a review of radiology reports. The nonradiographic component of SINS was the presence of mechanical back pain, which was obtained through the medical chart review. The total score was then calculated, and patients were assigned into one of three categories: stable (0-6 points), potentially unstable (7-12 points), and unstable (13-18 points). ${ }^{7}$ Statistical analysis for the SINS was performed as both continuous SINS (0, 1, 2, 3, etc.) and categorical SINS (0-6 vs 7-12 vs 13-18). For patients with multiple levels of metastasis, the lesion with the highest SINS was selected as the index level.

Predictors of VCF were identified by first performing univariable analysis between the variables and the presence of a new VCF within the reviewed time period, with the significance level set at $\mathrm{p}<0.05$. Univariable linear regression was performed for continuous variables and the chi-square test was used to analyze categorical variables. Variables shown to be significant with the univariable analysis were then examined in a multivariable logistic regression with a new VCF as the primary outcome. A univariable subanalysis was performed to identify factors associated with the VBQ score, and these results are shown in Supplemental Table 2. Values found on the multivariable analysis to have $\mathrm{p}<0.05$ were considered statistically significant. Receiver operating characteristics were calculated using logistic regression for the categorical SINS, the binary VBQ score, and the combination of both scores. JMP Pro 13 (SAS Institute Inc.) was used for all analyses.

\section{Results}

\section{Patient and Treatment Characteristics}

Of the 290 patients who received SBRT or surgical intervention for spinal metastasis during the reviewed period, 105 were included in this study. The preoperative demographics of these patients are summarized in Table 1 . There was a higher proportion of active smokers in the VCF group (12.5\% vs $2.0 \%, \mathrm{p}=0.023)$, as well as a greater proportion of patients who were previously treated with steroid medications for longer than 3 months $(48.2 \%$ vs $28.6 \%, \mathrm{p}=0.038)$. There were no other significant differences in patient demographics or previous treatments between these two groups. The mean follow-up time af- 
TABLE 1. Characteristics of 105 patients

\begin{tabular}{|c|c|c|c|}
\hline & Non-VCF Group (49 patients) & VCF Group (56 patients) & $p$ Value \\
\hline \multicolumn{4}{|l|}{ Demographics \& risk factors } \\
\hline Male & $26(53.1)$ & $31(55.4)$ & 0.814 \\
\hline Mean age, yrs & 62.0 & 60.5 & 0.482 \\
\hline Mean body mass index & 27.7 & 27.6 & 0.870 \\
\hline \multicolumn{4}{|l|}{ Smoking status } \\
\hline Never a smoker & $21(42.9)$ & $30(53.6)$ & \multirow{3}{*}{0.023} \\
\hline Former smoker & $27(55.1)$ & $19(33.9)$ & \\
\hline Current smoker & $1(2.0)$ & $7(12.5)$ & \\
\hline History of alcohol abuse & $2(4.1)$ & $1(1.8)$ & 0.479 \\
\hline Steroid use $>3$ mos & $14(28.6)$ & $27(48.2)$ & 0.038 \\
\hline NSAID use $>3$ mos & $7(14.3)$ & $9(16.1)$ & 0.799 \\
\hline Mean $\mathrm{CCl}$ & 8.1 & 7.9 & 0.563 \\
\hline KPS score & 85.4 & 85.2 & 0.938 \\
\hline \multicolumn{4}{|l|}{ ECOG Performance Status grade } \\
\hline 0 & $25(51.0)$ & $23(41.1)$ & \multirow{5}{*}{0.730} \\
\hline 1 & $20(40.8)$ & $28(50.0)$ & \\
\hline 2 & $3(6.1)$ & $3(5.36)$ & \\
\hline 3 & $1(2.0)$ & $2(3.6)$ & \\
\hline 4 & $0(0)$ & $0(0)$ & \\
\hline \multicolumn{4}{|l|}{ ASIA classification } \\
\hline$A / B$ & $0(0)$ & $1(1.8)$ & \multirow{4}{*}{0.724} \\
\hline C & $2(4.1)$ & $2(3.6)$ & \\
\hline $\mathrm{D}$ & $9(18.4)$ & $11(19.6)$ & \\
\hline$E$ & $38(77.6)$ & $43(76.8)$ & \\
\hline \multicolumn{4}{|l|}{ Laboratory value } \\
\hline Albumin (g/dl) & 4.2 & 3.9 & 0.111 \\
\hline Alkaline phosphatase (unit/L) & 104.8 & 128.5 & 0.380 \\
\hline Calcium & 9.2 & 9.1 & 0.759 \\
\hline Platelets $\left(\times 10^{3} / \mu \mathrm{L}\right)$ & 204.4 & 230.5 & 0.084 \\
\hline \multicolumn{4}{|l|}{ Previous VCFs \& treatment } \\
\hline Previous VCF & $10(20.4)$ & $16(28.6)$ & 0.332 \\
\hline Previous vertebroplasty/kyphoplasty & $0(0)$ & $3(5.4)$ & 0.100 \\
\hline Denosumab & $15(30.6)$ & $16(28.6)$ & 0.819 \\
\hline Cumulative radiation dose (cGy) & 2384.1 & 2584.2 & 0.152 \\
\hline Bisphosphonates & $12(24.5)$ & $17(30.4)$ & 0.501 \\
\hline Selective estrogen receptor modulator & $5(10.2)$ & $5(8.9)$ & 0.824 \\
\hline Calcium supplementation & $31(63.3)$ & $32(57.1)$ & 0.523 \\
\hline Vitamin D supplementation & $32(65.3)$ & $29(51.8)$ & 0.160 \\
\hline Systemic treatment duration (mos) & 32.1 & 24.6 & 0.164 \\
\hline Follow-up since spine metastasis diagnosis (mos) & 33.7 & 26.1 & 0.071 \\
\hline SINS & 5.0 & 7.1 & $<0.0001$ \\
\hline 1-6 (stable) & $40(81.6)$ & $20(35.7)$ & \multirow{3}{*}{$<0.0001$} \\
\hline 7-12 (potentially unstable) & $9(18.4)$ & $36(64.3)$ & \\
\hline 13-18 (unstable) & $0(0)$ & $0(0)$ & \\
\hline VBQ score & 2.48 & 3.26 & $<0.0001$ \\
\hline VBQ score $>3$ & $10(20.4)$ & $40(71.4)$ & $<0.0001$ \\
\hline
\end{tabular}

ASIA = American Spinal Injury Association; CCI = Charlson Comorbidity Index; ECOG = Eastern Cooperative Oncology Grade; KPS = Karnofsky Performance Scale.

Unless otherwise specified, values are expressed as number (\%) of patients. Boldface type indicates statistical significance. 
TABLE 2. Multivariate analysis of predictors of VCF

\begin{tabular}{lccc}
\hline \multicolumn{1}{c}{ Variable } & Estimate & $95 \% \mathrm{Cl}$ & $\mathrm{p} \mathrm{Value}$ \\
\hline VBQ score (continuous) & 3.051 & $1.860-4.601$ & $<0.0001$ \\
\hline SINS (continuous) & 0.879 & $0.509-1.368$ & $<0.0001$ \\
\hline Smoking history & - & - & 0.066 \\
\hline Prior steroid use $>3$ mos & - & - & 0.196 \\
\hline
\end{tabular}

Boldface type indicates statistical significance $(p<0.05)$.

ter the initial presentation of spinal metastases was 26.1 months for patients in the VCF group and 33.7 months for patients in the non-VCF group.

\section{Predictors of Vertebral Compression Fractures}

After the initial presentation with spinal metastases, 56 (53.3\%) of the 105 patients had one or more new VCFs at any vertebral level during the observation period. Table 2 illustrates the degree to which the SINS and VBQ score were predictive of the occurrence of subsequent VCFs. Patients in whom a VCF developed during the follow-up period had a significantly higher SINS at the initial presentation than patients with no new fracture ( 7.1 vs $5.0, \mathrm{p}$ $<0.0001)$. While none of the patients had a vertebra classified as "unstable" by the SINS criteria at the time their spinal disease was discovered, a significantly higher proportion of patients who subsequently experienced a VCF had a "potentially unstable" vertebra at diagnosis $(64.3 \%$ vs $18.4 \%, \mathrm{p}<0.0001)$. Patients with a VCF also had significantly higher VBQ scores at baseline than patients without a VCF (3.26 vs 2.48, p < 0.0001), with $71.4 \%$ of patients with fractures having a VBQ score exceeding the cutoff value of 3 and only $20.4 \%$ of patients without fractures having such a VBQ score $(\mathrm{p}<0.0001)$. Furthermore, it was found that patients with multiple VCFs had an even greater VBQ score, on average, than those with one or no VCF (3.40 vs $2.80, p<0.01)$.

Other significant predictors of VCF on the univariable analysis were smoking history $(\mathrm{p}<0.023)$ and steroid use for longer than 3 months ( $<<0.038$ ). However, on the multivariable analysis, only the SINS (OR 0.88 per point; $95 \%$ CI $0.509-1.368 ; \mathrm{p}<0.0001$ ) and the VBQ score (OR 3.05 per point; 95\% CI 1.860-4.601; $\mathrm{p}<0.0001$ ) were significantly associated with a new VCF, whereas smoking history and prior steroid use were not (Table 2).

\section{Evaluation of Predictive Scoring Measures}

Receiver operating characteristics for the categorical

TABLE 3. Outcome parameters of prediction scores for outcome of new VCFs

\begin{tabular}{lcccc}
\hline \multicolumn{1}{c}{ Score } & Sensitivity (\%) & Specificity (\%) & AUC & p Value \\
\hline Categorical SINS & 64.3 & 81.6 & 0.73 & $<0.0001$ \\
\hline VBQ score $>3$ & 75.0 & 85.7 & 0.80 & $<0.0001$ \\
\hline Combined & - & - & 0.89 & $<0.0001$ \\
\hline
\end{tabular}

Boldface type indicates statistical significance.

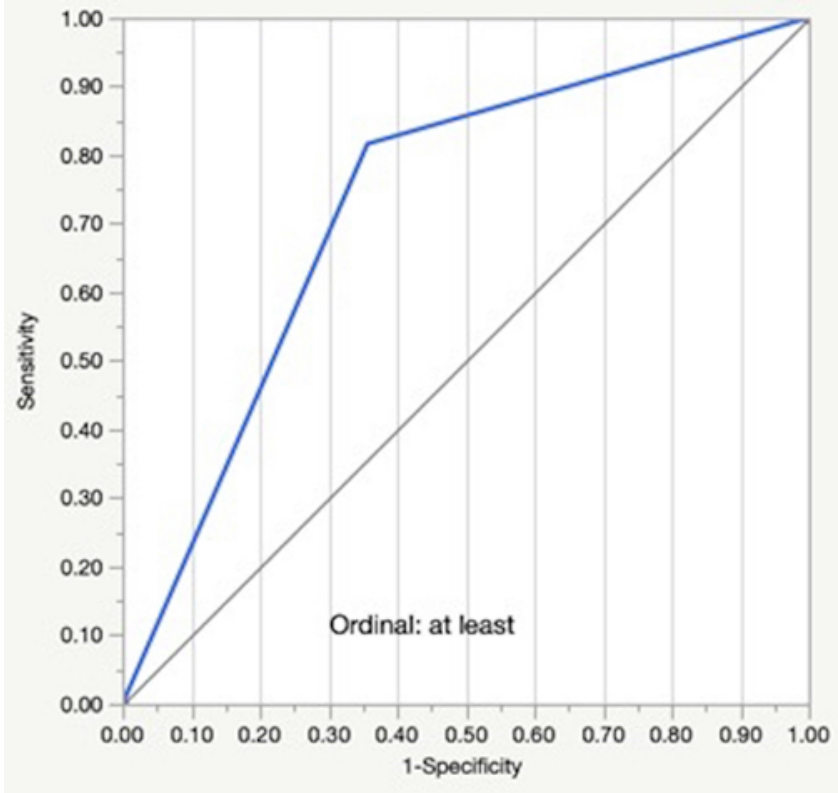

FIG. 2. Graph showing the AUC using the categorical SINS to predict a new VCF (AUC 0.73). Figure is available in color online only.

SINS, the categorical VBQ score, and the combination of the two scoring systems are summarized in Table 3. The categorical SINS predicted VCFs with a sensitivity of $64.3 \%$, a specificity of $81.6 \%$, and an area under the receiver operating characteristic curve (AUC) of 0.73 (Fig. 2). Using a VBQ score cutoff value of 3 , the categorical VBQ score yielded a sensitivity of $75.0 \%$ and a specificity of $85.7 \%$ with an AUC of 0.80 (Fig. 3). The VBQ score was shown to have excellent interrater reliability with an ICC of 0.899. Finally, the combined categorical SINS and binary VBQ score demonstrated the highest AUC of 0.89 (Fig. 4).

\section{Discussion}

In this paper, we describe the design of a novel MRIbased score for evaluating the global vertebral column bone quality in patients with metastatic spine disease. We find that this new score-the VBQ score-is significantly associated with the risk of VCF and predicts the occurrence of a VCF independent of the previously described SINS.

Growth of vertebral column metastases results in progressive destruction of the vertebral trabecular bone. Previous biomechanical studies have demonstrated that lesion size directly correlates to the biomechanical stability of the affected vertebra, as larger lesions produce vertebrae with significantly lower yield strengths. ${ }^{5,6,15,26}$ Clinically, this has been shown to translate to the risk of VCF. In their series of 53 patients with 100 metastatic lesions of the thoracolumbar spine, Taneichi et al. found that the proportion of vertebral body occupancy was the biggest predictor of a subsequent VCF. ${ }^{23}$ Because of this relationship, the SINS incorporates the extent of vertebral body involvement as one of its six components.

Although the SINS has been demonstrated to correlate 


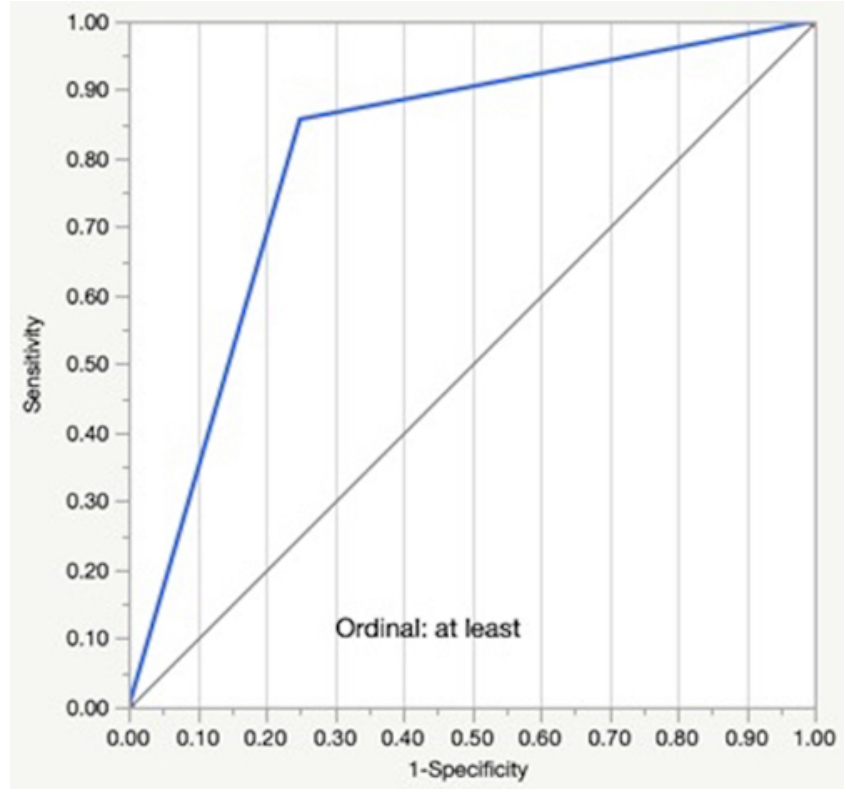

FIG. 3. Graph showing the AUC using a VBQ score $>3$ to predict a new VCF (AUC 0.80). Figure is available in color online only.

with the risk of $\mathrm{VCF},{ }^{22}$ it is imperfect as a predictive tool. The SINS focuses almost exclusively on the morphology of the spinal metastasis; it incorporates no description of baseline bone quality, which is also an important determinant of the overall biomechanical stability of the affected segment. Within the degenerative spine patient population, this latter metric is usually measured using DXA scan scores-based on a radiograph-based study looking at the extent of attenuation as a measure of BMD. ${ }^{14}$ However, DXA studies require a moderate degree of preparation and, for many clinicians, are not acquired as part of the standard workup for patients with metastatic spine disease. In the present study, we developed an MRI-based measure of baseline bone quality (the VBQ score) and demonstrated how it can independently predict VCF.

Several previous studies have also focused on creating an MRI-based means of quantifying bone quality (Mscore) with the goal of reducing patient radiation exposure and overall healthcare costs. ${ }^{1}$ MRI of trabecular bone takes advantage of the different degrees of signal intensity produced by the different components of the bone marrow. The three main factors affecting the MR signal intensity in bone marrow are the fat and water content, the presence of trabecular bone, and the contrast material that is used. ${ }^{24}$ Bone marrow can be separated into red and yellow marrow. Red marrow is often seen in "healthier" patients and is of a higher density; it thus provides a stronger structure. ${ }^{17}$ Yellow marrow has less strength due to its lower density; however, as lipid protons have shorter T1 relaxion times, they provide higher signal intensities on MRI, which can be taken advantage of by the current study.,16 Similarly, trabecular bone that contributes to overall vertebral strength produces very little signal intensity on MRI due to the lack of mobile protons.9 Therefore, "healthier" bone will be more hypointense on T1-weighted MRI due to greater trabecular bone and red marrow. ${ }^{2}$ However, sites

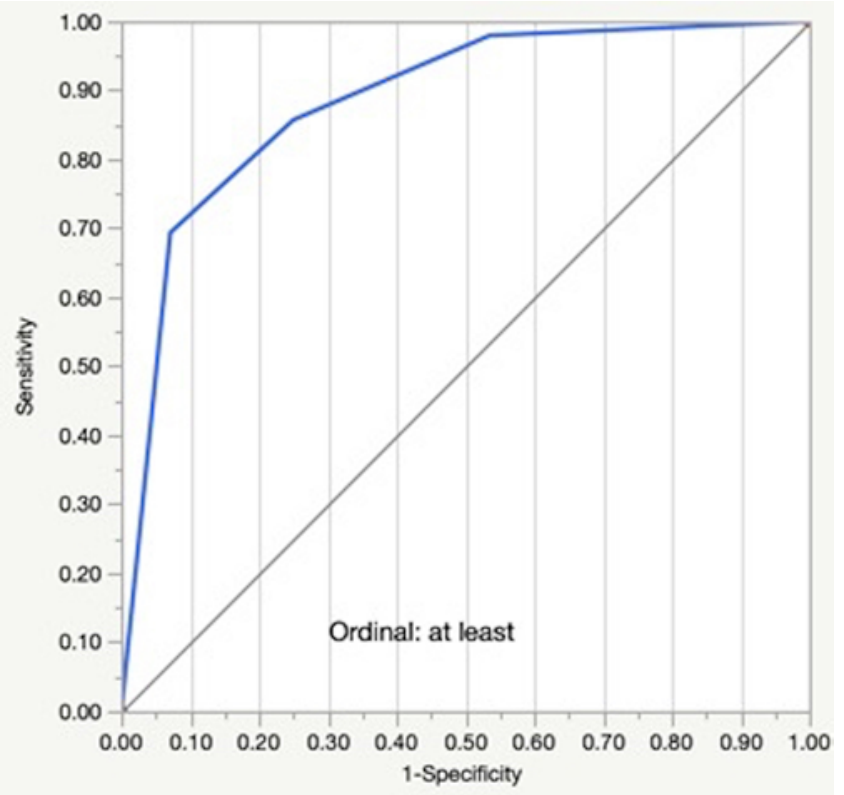

FIG. 4. Graph showing the AUC using a combined VBQ score $>3$ plus the categorical SINS to predict a new VCF (AUC 0.89). Figure is available in color online only.

of metastasis are also hypointense on T1-weighted MRI, so these locations must be excluded to avoid a confounded signal intensity measurement. ${ }^{9}$

Despite the potential utility of the M-score, it is limited by the fact that it relies on signal-to-noise ratios that are specific to the MR system in use. ${ }^{1,20}$ The original description by Bandirali et al. noted that this limitation of the Mscore would require each center to identify its own set of normalized values, an arduous task that potentially limits the clinical utility of this score. ${ }^{1}$ By contrast, the VBQ score described in the current study was implemented using MR volumes acquired by four distinct MR systems with no significant difference in VBQ scores between machines (Supplemental Table 1). Furthermore, the study by Bandirali et al. required a specific imaging protocol with set repetition and echo times, whereas the current study included all non-contrast-enhanced T1-weighted images, with the exception of inversion recovery images, which were excluded because of their suppression of the CSF signal. Therefore, the VBQ score maintains the diagnostic utility of the early M-score while having greater generalizability and potentially greater clinical utility.

In addition to the VBQ score and SINS, smoking and extended steroid use were also found to be associated with $\mathrm{VCF}$ in the univariable analysis. Based on data given in Supplemental Table 2, it is likely that the VBQ score accounted for bone changes from these two risk factors, therefore diminishing their trending significance in the multivariable analysis. However, it is important to discuss these factors, as they are modifiable. Lorentzon et al. found that smokers had significantly decreased trabecular bone density and thus lower bone strength. Interestingly, no change in bone density was discovered in cortical bone. ${ }^{13}$ Therefore, it is imperative to analyze trabecular bone, as in the current study, to truly understand the effects of smok- 
ing on overall bone strength. Similarly, it is well established that long-term corticosteroid use can induce osteoporosis and lead to fractures. ${ }^{19}$ Interestingly, previous authors have found that corticosteroid-induced osteoporosis often is evident initially in the high trabecular bone area of the lumbar spine. ${ }^{19}$ This again points to the importance of analyzing trabecular bone, as in the current study, in order to obtain an accurate estimate of current bone quality and provide patients with proper treatment to reduce future fractures.

The present study has limitations that should be considered. This study was retrospective in design, so a selection bias was possible when selecting which patients would undergo MRI versus CT or x-ray examination. Another limitation was the lack of DXA data in $90 \%$ of our patients, which necessitated that these data not be included in the present study. Although this highlights our point of DXA unavailability, it remains a limitation, as we were unable to test whether a correlation exists between the DXA and VBQ scores. Furthermore, it is not exactly clear whether the primary force driving the VCFs was osteoporosis or the metastatic elements in the vertebrae. Based on our findings that both the SINS and VBQ score were highly significant, it is likely a combination of the two factors. Another limitation is the potential for missed fractures, since the average follow-up time was less than 3 years. Patients who chose follow-up at outside institutions may have undergone less frequent imaging, thereby creating the risk of missing asymptomatic fractures. Finally, our method of calculating the signal intensity of lumbar vertebrae is simple compared to the MR spectroscopy method of quantifying bone marrow fat. ${ }^{3}$ It is possible that MR spectroscopy is better at avoiding radiographic limitations such as partial volume effects than our method of measuring from T1-weighted images. ${ }^{8}$ However, it is this simplicity of the VBQ score calculation, in addition to its demonstrated predictive power, that we believe makes this a tool with high potential to translate into clinical practice. To confirm our findings, external validation studies of the VBQ score are necessary, preferably across multiple institutions, MR systems, and patient populations.

\section{Conclusions}

This is the first study to evaluate the prognostic utility of an MRI-based measure of trabecular bone quality for predicting the occurrence of VCFs in patients with metastatic spine disease. We found that this MRI-based measure was a good predictor of VCFs, with a diagnostic accuracy of $80 \%$ for a cutoff score of 3 . More importantly, it was able to predict VCFs independent of the SINS, suggesting that baseline bone quality may predict VCFs independent of the extent of tumor-mediated bone destruction. We believe this study will hopefully spur future investigations into the role that baseline bone quality plays in influencing biomechanical outcomes in patients with metastatic spine disease.

\section{References}

1. Bandirali M, Di Leo G, Papini GD, Messina C, Sconfienza LM, Ulivieri FM, et al: A new diagnostic score to detect osteoporosis in patients undergoing lumbar spine MRI. Eur Radiol 25:2951-2959, 2015

2. Bloem JL, Reijnierse M, Huizinga TWJ, van der Helm-van Mil AHM. MR signal intensity: staying on the bright side in MR image interpretation. RMD Open 4:e000728, 2018

3. Cordes C, Baum T, Dieckmeyer M, Ruschke S, Diefenbach MN, Hauner H, et al: MR-based assessment of bone marrow fat in osteoporosis, diabetes, and obesity. Front Endocrinol (Lausanne) 7:74, 2016

4. Curry SJ, Krist AH, Owens DK, Barry MJ, Caughey AB, Davidson KW, et al: Screening for osteoporosis to prevent fractures: US Preventive Services Task Force Recommendation Statement. JAMA 319:2521-2531, 2018

5. Dimar JR II, Voor MJ, Zhang YM, Glassman SD: A human cadaver model for determination of pathologic fracture threshold resulting from tumorous destruction of the vertebral body. Spine (Phila Pa 1976) 23:1209-1214, 1998

6. Ebihara H, Ito M, Abumi K, Taneichi H, Kotani Y, Minami A, et al: A biomechanical analysis of metastatic vertebral collapse of the thoracic spine: a sheep model study. Spine (Phila Pa 1976) 29:994-999, 2004

7. Fisher CG, DiPaola CP, Ryken TC, Bilsky MH, Shaffrey CI, Berven $\mathrm{SH}$, et al: A novel classification system for spinal instability in neoplastic disease: an evidence-based approach and expert consensus from the Spine Oncology Study Group. Spine (Phila Pa 1976) 35:E1221-E1229, 2010

8. Hu HH, Kan HE: Quantitative proton MR techniques for measuring fat. NMR Biomed 26:1609-1629, 2013

9. Hwang S, Panicek DM: Magnetic resonance imaging of bone marrow in oncology, Part 1. Skeletal Radiol 36:913-920, 2007

10. Kanis JA, Cooper C, Rizzoli R, Reginster JY: Review of the guideline of the American College of Physicians on the treatment of osteoporosis. Osteoporos Int 29:1505-1510, 2018

11. Karampinos DC, Ruschke S, Gordijenko O, Grande Garcia E, Kooijman H, Burgkart R, et al: Association of MRS-based vertebral bone marrow fat fraction with bone strength in a human in vitro model. J Osteoporos 2015:152349, 2015

12. Lee BG, Choi JH, Kim DY, Choi WR, Lee SG, Kang CN: Risk factors for newly developed osteoporotic vertebral compression fractures following treatment for osteoporotic vertebral compression fractures. Spine J 19:301-305, 2019

13. Lorentzon M, Mellström D, Haug E, Ohlsson C: Smoking is associated with lower bone mineral density and reduced cortical thickness in young men. J Clin Endocrinol Metab 92:497-503, 2007

14. McCoy S, Tundo F, Chidambaram S, Baaj AA: Clinical considerations for spinal surgery in the osteoporotic patient: a comprehensive review. Clin Neurol Neurosurg 180:40-47, 2019

15. McGowan DP, Hipp JA, Takeuchi T, White AA III, Hayes WC: Strength reductions from trabecular destruction within thoracic vertebrae. J Spinal Disord 6:130-136, 1993

16. Meunier P, Aaron J, Edouard C, Vignon G: Osteoporosis and the replacement of cell populations of the marrow by adipose tissue. A quantitative study of 84 iliac bone biopsies. Clin Orthop Relat Res 80:147-154, 1971

17. Nishida Y, Matsue Y, Suehara Y, Fukumoto K, Fujisawa M, Takeuchi M, et al: Clinical and prognostic significance of bone marrow abnormalities in the appendicular skeleton detected by low-dose whole-body multidetector computed tomography in patients with multiple myeloma. Blood Cancer J 5:e329, 2015

18. Nouh MR, Eid AF: Magnetic resonance imaging of the spinal marrow: basic understanding of the normal marrow pattern and its variant. World J Radiol 7:448-458, 2015

19. Sambrook PN: How to prevent steroid induced osteoporosis. Ann Rheum Dis 64:176-178, 2005 
20. Shayganfar A, Khodayi M, Ebrahimian S, Tabrizi Z: Quantitative diagnosis of osteoporosis using lumbar spine signal intensity in magnetic resonance imaging. Br J Radiol 92:20180774, 2019

21. Shen W, Scherzer R, Gantz M, Chen J, Punyanitya M, Lewis $\mathrm{CE}$, et al: Relationship between MRI-measured bone marrow adipose tissue and hip and spine bone mineral density in African-American and Caucasian participants: the CARDIA study. J Clin Endocrinol Metab 97:1337-1346, 2012

22. Shi DD, Hertan LM, Lam TC, Skamene S, Chi JH, Groff M, et al: Assessing the utility of the spinal instability neoplastic score (SINS) to predict fracture after conventional radiation therapy (RT) for spinal metastases. Pract Radiat Oncol 8:e285-e294, 2018

23. Taneichi H, Kaneda K, Takeda N, Abumi K, Satoh S: Risk factors and probability of vertebral body collapse in metastases of the thoracic and lumbar spine. Spine (Phila Pa 1976) 22:239-245, 1997

24. Vanel D, Dromain C, Tardivon A: MRI of bone marrow disorders. Eur Radiol 10:224-229, 2000

25. Vlieger EJ, Maas M, Akkerman EM, Hollak CE, Den Heeten GJ: Vertebra disc ratio as a parameter for bone marrow involvement and its application in Gaucher disease. J Comput Assist Tomogr 26:843-848, 2002

26. Whyne CM, Hu SS, Lotz JC: Burst fracture in the metastatically involved spine: development, validation, and parametric analysis of a three-dimensional poroelastic finite-element model. Spine (Phila Pa 1976) 28:652-660, 2003

\section{Disclosures}

Dr. Khan reports being a consultant for Stryker Corporation and Medwaves Incorporated. Dr. Redmond reports having received research support, travel expenses, and/or honorarium for a speaking engagement, with respect to the Data and Safety Monitoring Board, from Accuray, Elekta AB, Brainlab, and Biometix. Dr. Sciubba reports being a consultant to Baxter, DePuy-Synthes, Globus Medical, K2M, Medtronic, NuVasive, and Stryker.

\section{Author Contributions}

Conception and design: Sciubba, Ehresman, Schilling, Gui, Chen, Redmond. Acquisition of data: Sciubba, Ehresman, Schilling, Ahmed, Redmond. Analysis and interpretation of data: Ehresman, Schilling, Pennington, Gui, Chen, Ahmed, Khan. Drafting the article: Ehresman, Schilling, Pennington, Lubelski, Cottrill. Critically revising the article: Sciubba, Pennington, Gui, Chen, Lubelski, Cottrill, Khan, Redmond. Reviewed submitted version of manuscript: Sciubba, Ehresman, Schilling, Chen, Lubelski, Ahmed, Cottrill, Khan, Redmond. Approved the final version of the manuscript on behalf of all authors: Sciubba. Statistical analysis: Ehresman.

\section{Supplemental Information \\ Online-Only Content}

Supplemental material is available with the online version of the article.

Supplemental Tables 1 and 2. https://thejns.org/doi/suppl/10. 3171/2019.9.SPINE19954.

\section{Correspondence}

Daniel M. Sciubba: Johns Hopkins University School of Medicine, Baltimore, MD. dsciubb1@jhmi.edu. 